\title{
Treatment Outcomes and Survival Study of Gastric Cancer Patients: A Retrospective Analysis in an Endemic Region
}

\author{
Hamit Basaran ${ }^{1}$, Timur Koca ${ }^{1 *}$, Arda Kaymak Cerkesli ${ }^{1}$, Deniz Arslan², Sibel \\ Karaca $^{1}$
}

\begin{abstract}
Purpose: To present information about prognostic factors of gastric cancer patients treated in our Erzurum center including age, gender, tumour location, pathological grade, stage and the effect of treatment on survival. Materials and Methods: This retrospective study was performed on patients who applied to our clinic and diagnosed as gastric cancer. Age and gender of the patients, primary location, histopathological characteristics, TNM stage of the gastric cancers (GCs), treatment applied, oncological treatment modalities and survival outcomes were studied. A univariate analysis of potential prognostic factors was performed with the log-rank test for categorical factors and parameters with a $p$ value $<0.05$ at the univariate step were included in the multivariate regression. Results: A total of 228 patients with a confirmed diagnosis of gastric cancer were included in the study with a male/female ratio of 1.47. Median follow-up period was estimated as 22.3 (range, 3 to 96) months. When diagnosis of the patients at admission was analysed, stage III patients were most frequently encountered $(n=147 ; 64.5 \%)$. One hundred and twenty-six $(55.3 \%)$ underwent surgical treatment, while 117 $(51.3 \%)$ were given adjuvant chemotherapy. Median overall survival time was $18.0( \pm 1.19)$ months. Mean overall survival rates for $1,2,3$ and 5 years were $68 \pm 0.031 \%, 36 \pm 0.033 \%, 24 \pm 0.031 \%$ and $15.5 \pm 0.036 \%$, respectively. Univariate variables found to be significant for median OS in the multivariate analysis were evaluated with Cox regression analysis. A significant difference was found among TNM stage groups, location of the tumour and postoperative adjuvant treatment receivers ( $p$ values were $0.011,0.025$ and 0.001 , respectively). Conclusions: This study revealed that it is possible to achieve long-term survival of gastric cancer with early diagnosis. Besides, in locally advanced GC patients, curative resection followed by adjuvant concomitant chemoradiotherapy based on the McDonald regimen was an independent prognostic factor for survival.
\end{abstract}

Keywords: Gastric cancer - overall survival - prognostic factors - chemoradiotherapy

Asian Pac J Cancer Prev, 16 (5), 2055-2060

\section{Introduction}

Gastric cancer (GC) is one of the most frequently seen type of cancer (Jemal et al., 2011). In recent years despite significant decreases in the incidence and mortality rates of GC, GC still continues to be an important public health issue worldwide (Yan et al., 2014). In the whole world it is a prevalent cancer type after lung, breast and colorectal cancers and every year 934,000 new cases have been diagnosed (Parkin et al., 2005). According to World Health Organization (WHO), gastric cancer is the most common cause of death (780.000 deaths/year) after lung cancer (Pisani et al., 1999; Zhu and Sonenberg., 2012). In Turkey it takes the 5th place among the most frequently seen cancer types (Seker et al., 2013). It is frequently seen during the 5th and 6th decades of life. Its median age of diagnosis is 65 years. Patients aged less than 40 years constitute $5-15 \%$ of all the diagnosed cases (Seker et al., 2013). In the year 2008, globally male/female ratio was calculated as 1.851 (Yu et al., 2014).

In the etiopathogenesis of gastric cancer, diet, environmental risk factors, socioeconomic status and Helicobacter pylori infection are implicated (Watanabe et al., 1998; Shikata et al., 2006; Tsugane et al., 2007; Tong et al., 2014). Among risk factors, especially living environment, food cooking and conservation methods and eating habits, especially consumption of diets deficient in fruits and green vegetables, but rich in nitrate and salty foods (Pleteiro et al., 2011), salted fish, smoked meat are among confirmed dietary risk factors (Dogan et al., 2009). The incidence of GC differs among geographic regions. Its incidence is higher in countries of Eastern Asia, Eastern Europe and Latin America and these regions are considered major endemic regions, while in Northern America and Northern Africa this disease is not frequently seen. This type of cancer in mostly $(>70 \%)$ in developing countries (Jemal et al., 2013; Elimova et al., 2014). Worldwide frequency of gastric 


\section{Hamit Basaran et al}

cancer has decreased within the last few decades. Its incidence also tends to decrease in Western Countries and Japan. In certain regions of the world its incidence is much higher and as an example to these regions Eastern Anatolian Region of Turkey which is an endemic region for upper gastrointestinal system tumours and western and northwestern regions of Iran can be indicated (Dogan et al., 2009; Alimoghaddam et al., 2014). Histopathological diagnosis of GC is adenocarcinoma in $90 \%$ of the cases (Seyedin et al., 2014). Currently, surgical treatment is the most effective treatment modality. R0 resection and D2 lymphadenectomy has been accepted as the standard treatment modalities (Wang et al., 2014; Zeng et al., 2014). Inclusion of adjuvant chemotherapy and radiotherapy in treatment protocol increases the success rate of treatment (Alimoghaddam et al., 2014; Misirlioglu et al., 2014; Zeng et al., 2014).

In this retrospective study, we present information about prognostic factors of gastric cancer patients treated in our center including age, gender, tumor location, pathological grade, stage and the effect of treatment on survival.

\section{Materials and Methods}

This retrospective study was performed on a total of 228 patients who applied to our clinic and diagnosed as gastric cancer in the Clinics of Medical Oncology and Radiation Oncology of Erzurum Regional Training and Research Hospital between September $5^{\text {th }}, 2006$ and June $30^{\text {th }}, 2014$. Data about the patients have been obtained by medical specialists via screening medical files and electronic data banks and from the patients and their relatives. Staging was based on the $7^{\text {th }}$ Edition of the Guideline of American Joint Committee on Cancer (AJCC) published in 2010.

Radiotherapy plans of 207 patients were performed using Cobalt-60 teletherapy device (Theratron, Elite 80 Model) and 2-dimensional anteroposterior radiation beam. However radiotherapy plans of 21 patients were realized with Linear accelerator device (Elekta, Synergy Model) using 3-dimensional conformal radiation therapy with a linear doses of equal distribution delivered from anterior, posterior, right and left lateral projections. For 145 patients who received curative radiotherapy, median dose (45 Gy; range, 44 to $54 \mathrm{~Gy})$ and median fractional dose (1.8 Gy; range, 1.8 to $2.0 \mathrm{~Gy}$ ) were also determined as indicated. For patients receiving palliative treatment median dose was detected as $30 \mathrm{~Gy} / 10$ fractions.

Patient with any metastatic disease were priorly evaluated regarding surgical indications. Definitive chemoradiotherapy (CRT) was recommended for patients who deemed to be inoperable by the Clinic of General Surgery. Preoperatively decision for postoperative CRT was given in collaboration with specialists in Medical Oncology and Radiation. During decision-making process for treatment, performance status and consent of the patients were also taken into consideration. Patients not amenable to curative treatment were included in palliative CT or RT programs.

Age and gender of the patients, primary location, histopathological characteristics, TNM stage of the GCs, treatment applied, oncological treatment modalities and survival outcomes are presented in Table 1.

Statistical Analyses: Data were analysed using the Statistical Package for Social Sciences 20.0 for Windows (SPSS Inc., Chicago, IL). Parametric tests were applied to data of normal distribution and non-parametric tests were applied to data of questionably normal distribution. Survival curves were estimated using the Kaplan-Meier method. A univariate analysis of potential prognostic factors was performed with the log-rank test for categorical factors and with the univariate Cox analysis for continuous variables. Data are expressed as mean \pm SD or median (interquartile range), as appropriate. All differences associated with a chance probability of .05 or less were considered statistically significant.

\section{Results}

A total of 228 patients with confirmed diagnosis of gastric cancer were included in the study. Demographic data of the patients are presented in Table 1. Median follow-up period was estimated as 22.3 (range, 3 to 96) months including the last follow-up period. At the end of the follow-up period, $167(73.2 \%)$ patients exited. Deceased cases consisted of $136(59.6 \%)$ male and 92 $(40.4 \%)$ patients with a male/female ratio of 1.47. Median overall survival time was 18.0 ( \pm 1.190$)$ months (Figure 1). Median OS estimated for male and female patients were $18 \pm 1.549$ and $17 \pm 1.848$ months, respectively without any statistically significant difference between both groups $(\mathrm{p}=0.857)$. Mean overall survival rates for $1,2,3$ and 5 years were $68 \pm 0.031 \%, 36 \pm 0.033 \%, 24 \pm 0.031 \%$ and $15.5 \pm 0.036 \%$, respectively.

The diagnoses of the patients were made when they were less $(n=135 ; 59.2 \%)$ and more $(n=93 ; 40.8 \%)$ than 65 years of age. Median age of the patients was 60.9 years and peak incidence of the disease was detected during the 7. decade of the patients' lifetime. In patients aged less and more than 65 years, median OS times were $20 \pm 1.808$ and $16 \pm 1.536$ months, respectively (Figure 2) with statistically significant difference between these two age groups $(\mathrm{p}=0.007)$.

Location of the tumours was evaluated based on endoscopic examinations, positron emission tomographycomputed tomography (PET-CT) findings and proximal tumors located on gastroesophageal junction (GEJ), cardia and fundus were compared with those detected on the body of stomach and distal stomach/antrum. Tumours located on proximal $(n=90 ; 39.5 \%)$, and distal $(n=68 ; 29.8 \%)$ parts of the stomach and on its body $(n=70 ; 30.7 \%)$ were also detected in respective percentages of the patients and corresponding median OSs were calculated as 17 , 34 and 17 months (Figure 4). Accordingly comparative results of the intergroup survival analysis were found to be statistically significant $(\mathrm{p}=0.001)$.

As histopathological diagnoses, adenocarcinomas (intestinal type) $(n=177)$, signet ring cell (diffuse type) carcinoma $(n=36)$ and in 15 cases other pathologies were detected. Overall survival rates did not differ as for histopathological diagnoses $(\mathrm{p}=0.520)$. 
Treatment Outcomes and Survival Study of Gastric Cancer Patients: A Retrospective Analysis in an Endemic Region Table 1. Patients Characteristics and Univariate Analysis of Survival for Prognostic Factors

\begin{tabular}{|c|c|c|c|c|c|c|c|}
\hline \multirow[t]{2}{*}{ Characteristics } & & \multicolumn{2}{|c|}{ Patients } & \multicolumn{2}{|c|}{ Events } & \multirow[t]{2}{*}{ Median OS (SD) } & \multirow[t]{2}{*}{$\mathrm{p}$ value } \\
\hline & & Number & $(\%)$ & Number & $(\%)$ & & \\
\hline \multirow[t]{2}{*}{ Gender } & Male & 136 & 59.6 & 103 & 61.7 & $18(1.549)$ & 0.857 \\
\hline & Female & 92 & 40.4 & 64 & 38.3 & $17(1.848)$ & \\
\hline \multirow[t]{2}{*}{ Age (year) } & $<65$ & 135 & 59.2 & 93 & 55.7 & $20(1.808)$ & 0.000 \\
\hline & $\geq 65$ & 93 & 40.8 & 74 & 44.3 & $16(1.536)$ & \\
\hline \multirow[t]{3}{*}{ Location } & Proximal (GEJ, Cardia, Fundus) & 90 & 39.5 & 74 & 44.3 & $17(0.963)$ & 0.001 \\
\hline & Body of Stomach & 70 & 30.7 & 56 & 33.5 & $17(1.369)$ & \\
\hline & Distalstomach, antrum & 68 & 29.8 & 37 & 22.2 & $34(10.231)$ & \\
\hline \multirow[t]{3}{*}{ Histopathology } & Adenocarcinoma(İntestinaltype) & 177 & 77.6 & 129 & 77.2 & $18(1.272)$ & 0.520 \\
\hline & Signet ring cell (Diffuse) & 36 & 15.8 & 26 & 15.6 & $14(1.988)$ & \\
\hline & Others & 15 & 6.6 & 12 & 7.2 & $22(5.973)$ & \\
\hline \multirow{3}{*}{ Pathologicalgrading } & Welldifferentiated & 31 & 13.6 & 18 & 10.8 & $19(4.526)$ & 0.015 \\
\hline & Moderatedifferentiated & 119 & 52.2 & 84 & 50.3 & $21(1.746)$ & \\
\hline & Poorlydifferentiated & 78 & 34.2 & 65 & 38.9 & $15(1.605)$ & \\
\hline \multirow[t]{4}{*}{ T stage } & $\mathrm{T} 1$ & 5 & 2.2 & 1 & 0.6 & -- & 0.137 \\
\hline & $\mathrm{T} 2$ & 11 & 4.8 & 8 & 4.8 & $30(4.404)$ & \\
\hline & $\mathrm{T} 3$ & 58 & 25.4 & 43 & 25.8 & $21(2.856)$ & \\
\hline & $\mathrm{T} 4$ & 154 & 67.6 & 115 & 68.8 & $17(0.924)$ & \\
\hline \multirow[t]{4}{*}{$\mathrm{N}$ stage } & No & 27 & 11.8 & 13 & 7.8 & $53(23.688)$ & 0.002 \\
\hline & N1 & 17 & 7.5 & 9 & 5.4 & $25(6.826)$ & \\
\hline & $\mathrm{N} 2$ & 52 & 22.8 & 40 & 24 & $17(2.062)$ & \\
\hline & N3 & 132 & 57.9 & 105 & 62.8 & $17(1.168)$ & \\
\hline \multirow{6}{*}{ TNM stage (AJCC 7th) } & $\mathrm{I}$ & 7 & 3.0 & 3 & 1.8 & -- & 0.000 \\
\hline & II & 30 & 13.2 & 16 & 9.6 & $25(7.541)$ & \\
\hline & IIIA & 26 & 11.4 & 18 & 10.7 & $27(7.810)$ & \\
\hline & IIIB & 54 & 23.7 & 39 & 23.4 & $19(1.724)$ & \\
\hline & IIIC & 67 & 29.4 & 51 & 30.5 & $18(2.341)$ & \\
\hline & IV & 44 & 19.3 & 40 & 24 & $10(1.893)$ & \\
\hline \multirow[t]{4}{*}{ Surgical Treatment Modality } & No operation & 102 & 44.7 & 88 & 52.7 & $13(1.218)$ & 0.000 \\
\hline & Total gastrectomy & 74 & 32.5 & 54 & 32.3 & $22(2.606)$ & \\
\hline & Proximalgastrectomy & 6 & 2.6 & 5 & 3.0 & $18(2.309)$ & \\
\hline & Distalgastrectomy & 46 & 20.2 & 20 & 12.0 & $65-$ & \\
\hline \multirow[t]{4}{*}{ Oncologic Treatment Modality } & Onlyoperation & 5 & 2.2 & 5 & 3.0 & $19(1.095)$ & 0.000 \\
\hline & Definitive RT/CRT & 51 & 22.4 & 44 & 26.3 & $15(0.565)$ & \\
\hline & Post operativeadjuvant CRT & 117 & 51.3 & 71 & 42.6 & $25(4.011)$ & \\
\hline & Palliation & 55 & 24.1 & 47 & 28.1 & $11(1.481)$ & \\
\hline \multirow[t]{4}{*}{ Chemotherapy (CT) } & FUFA & 118 & 77.6 & 76 & 74.5 & $24(4.646)$ & 0.001 \\
\hline & $5 \mathrm{FU}$ & 8 & 5.3 & 3 & 2.9 & $21-$ & \\
\hline & DCF & 19 & 12.5 & 17 & 16.6 & $16(1.957)$ & \\
\hline & $\mathrm{DC}$ & 7 & 4.6 & 6 & 6 & $14(5.237)$ & \\
\hline
\end{tabular}

Histopathological grades of the patients were reported as well $(n=31)$, moderately $(n=119)$ and poorly $(n=78)$ differentiated. Based on histopathological grading patients with well and moderately differentiated tumours did not demonstrate longer survival times when compared with those with poorly differentiated tumours $(\mathrm{p}=0.015)$.

Distribution of the patients who received treatment based on their TNM stages at the time of diagnosis was as follows: $\mathrm{T} 1(\mathrm{n}=5 ; 2.2 \%), \mathrm{T} 2(\mathrm{n}=11 ; 4.8 \%), \mathrm{T} 3(\mathrm{n}=58$; $25.4 \%)$ T4 $(n=154 ; 67.6 \%)$, N0 $(n=27 ; 11.8 \%), N 1$ $(\mathrm{n}=17 ; 7.5 \%), \mathrm{N} 2(\mathrm{n}=52 ; 25.8 \%), \mathrm{N} 3(\mathrm{n}=132 ; 57.9 \%)$ , M0 ( $\mathrm{n}=184 ; 80.7 \%)$, and M1 $(\mathrm{n}=44 ; 19.3 \%)$. When diagnosis of the patients at admission were analysed, Stage III patients were more frequently encountered $(n=147$; $64.5 \%)$ followed by Stage IV $(n=44 ; 19.3 \%)$, II $(n=30$; $13.2 \%)$ and $I(n=7 ; 3.1 \%)$ patients (Table 1$)$.

Evaluation of survival rates of the patients was based on TNM and clinical stages. Since Stage III patients consisted of large number of patients, these patients were analysed in subgroups of Stage IIIA, IIIB and IIIC with respective distribution rates of $11.4 \%, 23.7 \%$ and
29.4\%. Median overall survival rates did not statistically significantly differ as for $\mathrm{T}$ stages $(\mathrm{p}=0.137)$, however according to $\mathrm{N}(\mathrm{p}=0.001)$ and TNM $(\mathrm{p}=0.000)$ stages median OS rates differed statistically significantly (Figure 3).

A hundred and two (44.7\%) patients did not undergo surgical treatment, while the remaining patients underwent total gastrectomy $(\mathrm{n}=74 ; 32.5 \%)$, proximal gastrectomy $(\mathrm{n}=6 ; 2.6 \%)$ and distal gastrectomy $(\mathrm{n}=46 ; 20.2 \%)$. Median overall survival times in the non-surgical followup group , total, proximal and distal gastrectomy groups were $13,22,18$ and 65 months, respectively $(\mathrm{p}=0.00)$. Since five patients who received oncological treatment modality were in their postoperative early stage, they were followed up without adjuvant therapy. Fifty- one patients were treated conservatively with definitive RT/ CRT Postoperative adjuvant CRT therapy was performed on 117 (51.3\%) patients. Fifty-five patients with Stage IV or those whose general health state was not suitable for any other treatment modality received palliative therapy. The patients receive various CT protocols. However $88.9 \%$ of 


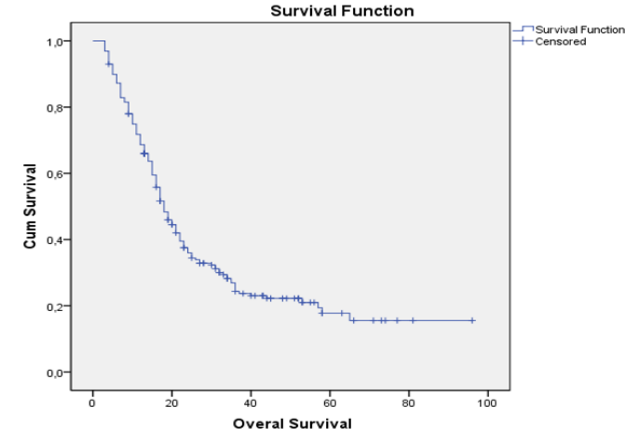

Figure 1. Overall Survival Curves of 228 Patients Treated for Gastric Cancer

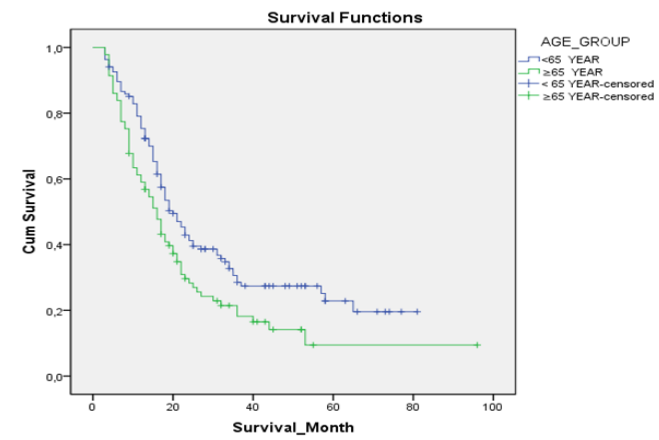

Figure 2. Overall Survival Curves of 228 Patients Treated for Gastric Cancer Based on Age Groups

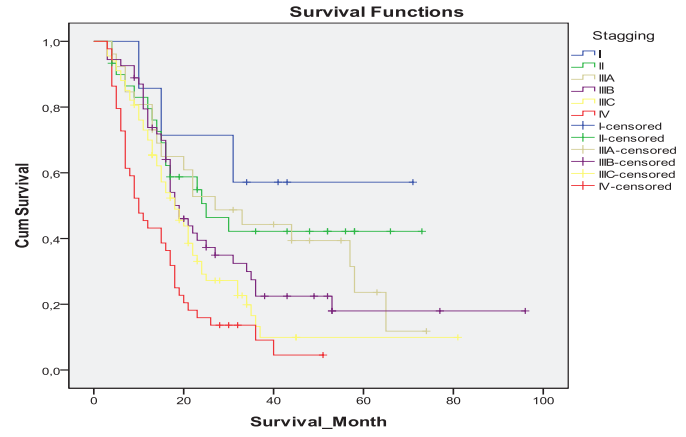

Figure 3. Survival Curves of 228 Patients Treated for Gastric Cancer According to AJCC 7th 2010 TNM Staging System

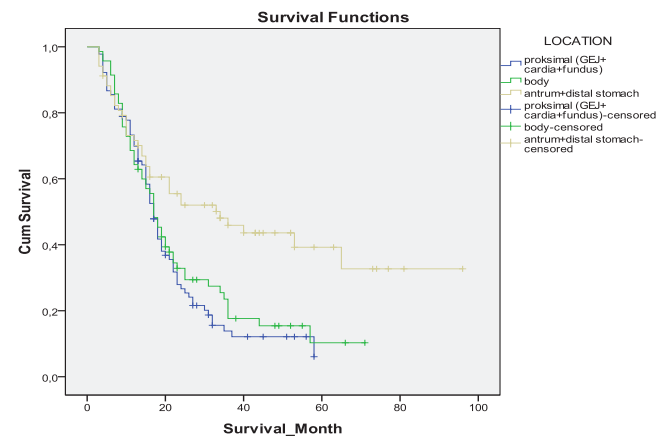

Figure 4. Comparison of Survival Curves between Patients with Various Tumor Location for Gastric Cancer

the patients who underwent postoperative CRT received FUFA (5-flourouacil+folinic acid) protocol based on the INT0116 /SWOG (McDonald et al.) Survival analyses
Table 2. Multivariateanalysis in Overallsurvival of Patientstreatedforgastriccancer

\begin{tabular}{llcccc}
\hline Variable & \multicolumn{3}{c}{$95.0 \%$ CI } & SD & p value \\
& & Lower & Upper & & \\
\hline TNM stage $(\mathrm{p}=0.011)$ & I & 0.080 & 0.860 & 0.607 & 0.027 \\
& II & 0.215 & 0.698 & 0.301 & 0.002 \\
& IIIA & 0.270 & 0.866 & 0.298 & 0.015 \\
& IIIB & 0.397 & 0.987 & 0.232 & 0.044 \\
& IIIC & 0.467 & 1,082 & 0.214 & 0.112 \\
Postoperativeadjuvant CRT & 0.631 & 0.893 & 0.089 & 0.001 \\
Pathologicalgrading & & 0.968 & 1,618 & 0.131 & 0.088 \\
Tumorlocation & & 0.650 & 0.972 & 0.103 & 0.025 \\
\hline
\end{tabular}

$\mathrm{CI}=$ Confidenceintervals; $\mathrm{SD}=\mathrm{Standard}$ Deviation ; $\mathrm{CRT}=$ Chemoradiotherapy

on oncological treatment modalities detected median survival times in patients followed up after surgery $(n=5)$ and in definitive RT/CRT, postoperative adjuvant CRT and palliative treatment groups were 19,15 and 11 months, respectively with statistically significant differences between groups $(\mathrm{p}=0.000)$.

Chemotherapy (CT) was performed in 152 patients. The most frequently used CT protocol was FUFA $(n=118$; $77.6 \%$ ). While patients also received DCF (Docetaxel, Cisplatin and 5-fluorouracil) $(\mathrm{n}=19 ; 12.5 \%), 5-\mathrm{FU}$ (5-flourouacil) $(\mathrm{n}=8 ; 5.3 \%)$ and DC (docetaxel-cisplatin) $(n=7 ; 4.6 \%)$ protocols. Median OS times for these CT protocols were calculated as 24,21, 16 and 14 months, respectively and differences between them were not found to be statistically significant $(\mathrm{p}=0.001)$.

Most of the cases $(n=222 ; 97.4 \%)$ with GC included in the study received radiotherapy at different stages of the disease. The patients also underwent curative RT $(n=166 ; 72.8 \%)$, palliative treatment $(n=54 ; 23.7 \%)$ and radiotherapy after recurrence $(n=2 ; 0.9 \%)$.

Univariate variables found to be significant for median OS in the multivariate analysis were evaluated with Cox regression analysis. A significant difference was found among TNM stage groups, location of the tumour and postoperative adjuvant treatment receivers ( $\mathrm{p}$ values were $0.011,0.025$ and 0.001 , respectively) (Table 2).

\section{Discussion}

Gastric cancer has a poor prognosis and 5-year overall survival rates range between 15 and 20 percent. However within the 30 years a slight but definitive improvement has been achieved (Bamias and Pavlidis, 1998).

Our study has revealed very important implications related to prognostic factors which presumably have an impact on characteristics and survival of the patients treated with the diagnosis of gastric cancer living in the Eastern Anatolian Region of Turkey where upper gastrointestinal system tumours are endemic. Various studies have demonstrated the significance of clinicopathological factors and especially age, gender, location, histopathological diagnosis, grade, TNM stage of the tumours, postoperative adjuvant chemotherapy and radiotherapy and their combinations on GC-specific OS (MacDonald et al., 2001; Yang et al., 2011; Lin et al., 2014; Seyedin et al., 2014). 
Mean age of our study population was 60.4 years with a male/female ratio (MFR) of 1.47. This ratio demonstrates differences among various countries. For instance MFR was reported as nearly 6:1 in the United States (Yan et al., 2014). In 2008, this ratio was estimated as 1.851 (Yu et al., 2014). In our study, our estimate is close to those reported in the literature. This difference between genders is not completely attributed to the prevalence of risk factors known to exist between genders. Besides a hypothesis asserts the protective role of hormones (ie. estrogens) in women. (Chandanos and Lagergren, 2008).

In our study survival difference was not detected between genders. However some studies have demonstrated that GC-specific mortality risk in women is lower relative to male patients (Yang et al., 2011).

In a study by Sup Kim et al., the cases were diagnosed as Stage IB-II (9\%), IIIA (71\%) and IV (21\%) patients with rates similar to those found in our patient groups (Kim et al., 2011).

In our study, corresponding 1-, 2- and 5-year survival rates were $79.5, \% 50.6$ and $42 \%$ in stage II, $73.3,37.4$ and $14 \%$ in stage III and $43.2,13.6$ and 0 in stage IV. In the literature, 5-year survival rates of the patients with adjuvant treatment indication and treated with McDonald regimen were $90 \%$ in stage II, $50 \%$ in stage III and $20 \%$ in stage IV (Selcukbiricik et al., 2013).

Most of the patients $(64.5 \%)$ were diagnosed in advanced clinical stage (stage III) of the disease. In these patients, gastric cancer is defined as regional and systemic disease, rather than a local disease (Zeng et al., 2014). Some (19.3\%) of the patients applied to the hospital while they were in stage IV and became candidates for palliative treatments. Only $16 \%$ of the patients were diagnosed during early stage of their disease. With early diagnosis, achievement of the chance of curative treatment and increasing survival rates appear to be a possibility.

In our study, at the end of the follow-up period 167 patients died. Seventy-one (42.5\%) patients died from cancer-related causes within the first year and 68 patients $(40.7 \%)$ died within the second year after establishment of diagnosis. When compared with the study by Weijung Zeng et al. mortality rates within the first year were similar, however in the second year mortality rates were higher. Most of the deaths from gastric cancer are thought to be related to the recurrence of GC (Zeng et al., 2014). Routine and regular carcinoembryonic antigen (CEA) and carboxyl antigen 19-9 (CA19-9) controls during the follow-up period, recognition of the recurrence during the early stage of the disease and optimal treatment play important roles in decreasing patients' mortality rates (Zeng et al., 2014).

Majority of the patients (51.3\%) was diagnosed as locally advanced disease and they received postoperative adjuvant CRT in compliance with INT 0116 study. When survival analysis of the treatment groups was examined, median OS rates were 15 months in the definitive RT/ CRT, 25 months in the postoperative adjuvant CRT and 11 months in the palliative treatment groups $(\mathrm{p}=0.000)$. In the treatment of locally advanced gastric cancer, postoperative adjuvant CRT has advantages of local control and overall survival which were previously demonstrated in a few randomized phase III studies (Kim et al., 2005; Mac Donald et al., 2001). In an INT 0116 study by McDonald et al., it has been demonstrated that addition of postoperative adjuvant CRT to surgery increased median survival time from 26 months up to 35 months $(\mathrm{p}=0.006)$ and 3 -year $\mathrm{OS}$ from 41 to $50 \%(\mathrm{p}=0.01)$. Later on Kim et al. confirmed the same outcomes in their case-controlled studies and their 5-year survival rates were always relatively higher in the CRT group (Kim et al., 2005; Kim et al., 2011).

Though many of our study patients were diagnosed and included in the treatment protocol during advanced clinical stage of their disease, our survival outcomes are comparable to those mentioned in the literature.

In conclusion, this study revealed important prognostic factors effective on the survivals of the patients diagnosed as GC. It is possible to achieve long-term survival with early diagnosis. Besides, in locally advanced GC patients, curative resection followed by adjuvant concomitant CRT based on McDonald regimen has been demonstrated as an independent prognostic factor of survival.

\section{References}

Alimoghaddam K, Jalali A, Aliabadi LS, et al (2014). The outcomes of esophageal and gastric cancer treatments in a retrospective study, single center experience. Int J Hematol Oncol Stem Cell Res, 8, 9-13.

Bamias A, Pavlidis N(1998). Systemic chemotherapy in gastric cancer: where do we stand today? Oncologist, 3, 171-7.

Chandanos E, Lagergren J(2008). Oestrogen and the enigmatic male predominance of gastric cancer. Eur J Cancer, 44, 2397-403.

Dogan NB, Timur K, Hakan D(2009). Dietary habits, demographical, and socio-economical risk factors of the newly diagnosed gastric cancers in the eastern anatolia region of turkey: an endemic upper gastrointestinal cancer region. Dig Dis Sci, 54, 2629-33.

Elimova E, Shiozaki H, Wadhwa R, et al (2014). Medical management of gastric cancer: A 2014 update. World $J$ Gastroenterol, 20, 13637-47.

Jemal A, Bray F, Center MM, et al (2011). Global cancer statistic. CA Cancer J Clin, 61, 69.

Kim S, Kim JS, Jeong HY, et al (2011). Retrospective analysis of treatment outcomes after postoperative chemoradiotherapy in advanced gastric cancer. Radiat Oncol J, 29, 252-9.

Kim S, Lim DH, Lee J, et al (2005). An observational study suggesting clinical benefit for adjuvant postoperative chemoradiation in a population of over 500 cases after gastric resection with D2 nodal dissection for adenocarcinoma of the stomach. Int J Radiat Oncol Biol Phys, 63, 1279-85.

Lin WL, Sun JL, Chang SC, et al (2014). Factors predicting survival of patients with gastric cancer. Asian Pac J Cancer Prev, 15, 5835-8.

Macdonald JS, Smalley SR, Benedetti J, et al (2001). Chemoradiotherapy after surgery compared with surgery alone for adenocarcinoma of the stomach or gastroesophageal junction. N Engl J Med, 345, 725-30.

Misirlioglu HC, Coskun-Breuneval M, Kucukpilakci B, et al (2014). Adjuvant radiotherapy for gastric carcinoma: 10 years follow-up of 244 cases from a single institution. Asian Pac J Cancer Prev, 15, 8871-6.

Parkin DM, Bray F, Ferlay J, et al (2005). Global cancer statistics, 2002. CA Cancer J Clin, 55, 74-108.

Peleteiro B, Lopes C, et al (2011). Salt intake and gastric cancer risk according to Helicobacter pylori infection, smoking, 
tumour site and histological type. Br J Cancer, 104, 198.

Pisani P, Parkin DM, Bray F, Feraly J (1999). Estimates of the worldwide mortality from 25 cancers in 1990 . Int J Cancer, 83, 18-29.

Seker M, Aksoy S, et al (2013). Clinicopathologic features of gastric cancer in young patients. Saudi J Gastroenterol, 19, 258-61.

Selcukbiricik F, Buyukunal E, Tural D, et al (2013). Clinicopathological features and outcomes of patients with gastric cancer: a single-center experience. World $J$ Gastroenterol, 19, 2154-61.

Seyedin S, Wang P, Zhang Q, Lee P(2014). Benefit of adjuvant chemoradiotherapy for gastric adenocarcinoma:a seer population analysis. Gastrointest Cancer Res, 7, 82-90.

Shikata K, Kiyohara Y, Kubo M, et al (2006). A prospective study of dietary salt intake and gastric cancer incidence in a defined Japanese population: the Hisayama study. Int $J$ Cancer, 119, 196.

Tong GX, Liang H, Chai J, et al (2014). Association of risk of gastric cancer and consumption of tobacco, alcohol and tea in the Chinese population. Asian Pac J Cancer Prev, 15, 8765-74.

Tsugane S, Sasazuki S, et al (2007). Diet and the risk of gastric cancer: review of epidemiological evidence. Gastric Cancer, 10,75 .

Wang Z, Ma L, Zhang XM, Zhou ZX(2014). Long-term outcomes after D2 gastrectomy for early gastric cancer: survival analysis of a single-center experience in China. Asian Pac J Cancer Prev, 15, 7219-22.

Watanabe T, Tada M, et al (1998). Helicobacter pylori infection induces gastric cancer in mongolian gerbils. Gastroenterology, 115, 642.

Yan S, Li B, Bai ZZ, et al (2014).Clinical epidemiology of gastric cancer in Hehuang valley of China: a 10 year epidemiological study of gastric cancer. World J Gastroenterol, 20, 10486-94.

Yang D, Hendifar A, Lenz C, et al (2011). Survival of metastatic gastric cancer: Significance of age, sex and race/ethnicity. $J$ Gastrointest Oncol, 2,77-84.

Yu J, He Y, Guo Z(2014). Age trend of the male to female sex ratio in surgical gastric cancer patients at a single institution. World J Surg Oncol, 12, 269.

Zeng WJ, Hu WQ ,Wang LW, et al (2014). Long term follow up and retrospective study on 533 gastric cancer cases. BMC Surg, 14, 29.

Zhu AL, Sonnenberg A(2012). Is gastric cance ragain rising? $J$ Clin Gastroenterol, 46, 804-6. 